\title{
Nanocrystal Diffusion in a Liquid Thin Film Observed by in situ Transmission \\ Electron Microscopy
}

Haimei Zheng ${ }^{1,2,3}$, Shelley A. Claridge ${ }^{3}$, Andrew M. Minor ${ }^{1,2,4}$, A. Paul Alivisatos ${ }^{2,3} *$, Ulrich Dahmen $^{1,2} \#$

${ }^{1}$ National Center for Electron Microcopy, Lawrence Berkeley National Laboratory, Berkeley, CA 94720, USA

${ }^{2}$ Materials Sciences Division, Lawrence Berkeley National Laboratory, Berkeley, CA 94720, USA

${ }^{3}$ Department of Chemistry, University of California, Berkeley, CA 94720, USA

${ }^{4}$ Department of Materials Science and Engineering, University of California, Berkeley, CA 94720, USA

We have directly observed motion of inorganic nanoparticles during fluid evaporation using a Transmission Electron Microscope. Tracking real-time diffusion of both spherical $(5-15 \mathrm{~nm})$ and rod-shaped $(5 \times 10 \mathrm{~nm})$ gold nanocrystals in a thin-film of water- $15 \%$ glycerol reveals complex movements, such as rolling motions coupled to large-step movements and macroscopic violations of the Stokes-Einstein relation for diffusion. As drying patches form during the final stages of evaporation, particle motion is dominated by the nearby retracting liquid front.

To whom correspondence should be addressed. *Email: alivis@berkeley.edu or \#Email: udahmen@1bl.gov. 
When a liquid that contains colloidal nanoparticles evaporates from a surface, a variety of intricate patterns can form ${ }^{1,2}$. In a controlled drying process ${ }^{3,4}$ large-scale arrays of highly organized patterns of nanoparticles can be generated. For example, capillary forces can overcome the random thermal fluctuations so that nanoparticles diffuse into pre-patterned holes or templates on a substrate surface ${ }^{4}$. Controlled selfassembly of nanocrystals into functional patterns holds promise as a scalable fabrication strategy to systematically produce nanoscale devices. However, fluid deposition of nanoparticles is poorly understood and generally not predictable at the present time. One of the fundamental questions underlying particle assembly is what are the characteristics of the particle diffusion near surfaces and during the last moments before liquid drying?

As the thickness of a solution approaches the nanometer scale, several factors influence the particle motion. These include solvent surface fluctuations ${ }^{2}$, airliquid $\backslash$ substrate-liquid interface structure ${ }^{4,5}$ as well as the intrinsic differences in the relaxation and transport properties in an ultra thin liquid film compared to its bulk ${ }^{6-9}$. Diffusion of nanoparticles in such thin liquid films is largely beyond the predictive capabilities of current theoretical computation ${ }^{10}$. The challenge for experimentalists is that it has not been possible to directly image the details of the dynamical diffusion processes in real time due to instrumental limitations.

By taking advantage of the high spatial resolution of a transmission electron microscope (TEM), we were able to observe the microscopic details of nanoparticle motion during fluid evaporation. Imaging of liquid samples using a TEM is achieved here by using a newly designed self-contained liquid cell (see details on the liquid cells in 
Supporting Information and related techniques by Williamson et al. ${ }^{12}$ ). For imaging, about a hundred nanoliters of a dilute solution of Au nanoparticles in a water-glycerol mixture was loaded into one of the reservoirs in the liquid cell. The solution was dilute in order to avoid interactions between the gold particles for this work, although concentrated solutions could also be examined by this method (see liquid sample preparation in Supporting Information). Liquid solution was drawn from the reservoir into the window by capillary forces and formed a liquid layer confined between two electron transparent silicon nitride membranes. Subsequently, the liquid cell was sealed and loaded into a TEM as a standard TEM sample.

The liquid slowly evaporates inside the microscope due to the imperfect seal of the cell in a vacuum environment and a relative high vapor pressure of the liquid. Consequently, one side of the liquid film generally detaches from the silicon nitride membrane, creating a vapor-liquid interface. Observations are thus of particles moving in a thin liquid film between a solid substrate and a liquid-vapor interface, as it would occur during most drying processes. Due to the slow evaporation rate of the fluid $(\sim 1 \mathrm{~nm} / \mathrm{min}$, see Supporting Information), our observations are of the particle motion in a liquid thin film with negligible changes in the film thickness.

We first study the nanoparticle motion before the formation of drying patches and when the liquid thickness is close to but greater than the nanoparticle diameter. Throughout this period, the nanoparticles execute a complex trajectory of motions which show significant effects from the substrate surface. From image analysis, we obtain data sets consisting of a particle's two-dimensional center-of-mass positions $R\left(t_{i}\right)=\left[x\left(t_{i}\right), y\left(t_{i}\right)\right]$ in the lab frame with spatial resolution of $1 \mathrm{~nm}$ and temporal 
resolution of 30 milliseconds. For asymmetric particles we also measure the orientation angles, $\theta\left(t_{i}\right)$, relative to the $x$-axis with resolution of $1^{\circ}$ (Fig. 3C). Microscopic details of the particle movement can be obtained from the individual video frames.

We have considered the effects that the electron beam might have on the particle motion, including local heating ${ }^{12,13}$, direct momentum transfer from the electron beam ${ }^{12}$, and electron charging ${ }^{14}$. In the water-glycerol mixture under study here, thermal fluctuations in the liquid are large compared to the energy imparted to the particles by the electron beam. Thus, while an energetic electron beam such as that in the TEM can drive nanoparticle motion in some circumstances, such as on a dry substrate, this is not a significant consideration here, as the electron beam effects on particle motion is a few orders of magnitude smaller than the liquid thermal effects. It is also evidenced by the negligible differences in particle trajectories obtained at beam currents that differ by a factor of five (see the theoretical calculation and experiments in Supporting Information).

Due to the complexity of a non-equilibrium liquid under evaporation, it is difficult to estimate the exact liquid conditions (e.g. liquid thickness, etc.), which makes it impractical to compare particle diffusion from different samples. In order to study the variation of particle motion as a function of nanoparticle size, we mixed nanoparticles of different sizes together, and collected trajectories from particles of different sizes that were close to each other. Trajectories of two-dimensional particle displacement for three particles sizes, 5, 10 and $15 \mathrm{~nm}$ in diameter, in the same liquid film recorded in the same time period before drying patches initiated are shown in Fig. 1A (also see movie S1). A liquid thickness of roughly 20-30 nm was estimated by assuming a linear evaporation rate $^{15}$. The particle trajectories exhibit sparse and larger step movements, which we term 
“jumps", between swarms of much smaller steps. Larger jump distances were observed for larger particles. In order to quantify this behavior, we analyzed the particle displacement $(\lambda)$ during a time interval $(\Delta t)$ as a function time $(t)$, shown in Fig. 1B (see detailed analysis in Supporting Information and reference by Raptis et al. ${ }^{16}$ ). Particle jumps, corresponding to peaks in the plot in Fig. 1B, are followed by an extended series of small-step displacements along the trajectory. There was no obvious correlation between the jumps of different, but nearby particles. Thus, we conclude that these jumps are not the result of large-scale liquid motion (for example, convection or turbulence), which would be similar for nearby particles within the small field of view (about 100 $\left.\mathrm{nm}^{2}\right)$. The mean-square displacement, $M S D\left(<x^{2}>\right)$, including all multi-scale step movements is approximately linear with time $(t)$, see Fig. 1E. This allows estimation of two-dimensional diffusion coefficients $D=<x^{2}>/ 4 t$, from which $0.165 \mathrm{~nm}^{2} / \mathrm{s}$ for the 5 $\mathrm{nm}, 0.172 \mathrm{~nm}^{2} / \mathrm{s}$ for the $10 \mathrm{~nm}$, and $0.268 \mathrm{~nm}^{2} / \mathrm{s}$ for the $15 \mathrm{~nm}$ particle were obtained. It is interesting to note that the larger diffusion coefficients observed for larger particles violates the Stokes-Einstein relationship, in which $D$ scales inversely with particle diameter.

Detailed characterization of the particle motion reveals the mechanism of this violation. The histograms of particle displacement distribution show multiple peaks corresponding to the different scales of step movements, namely, a main peak around zero displacement due to the small-step movements and subsidiary peaks due to the larger step movements (Fig. 1D). The main peaks around zero displacement can be fit by Gaussian distributions. The standard deviation of the distribution represents the average step displacement during the time interval. A diffusion constant due to the small-step 
movements only can be obtained from the average small-step displacement as a function of time. The diffusion constant due to the small-step movement is roughly inversely proportional to particle size, see Fig. 1E. Therefore, the larger step jump diffusion is the main contribution to the violation of the Stokes-Einstein relationship.

Analysis of individual images indicates particle contrast changes during each jump (see Fig. 2A and B, as examples). However, the particle contrast mostly remains the same for the small-step movements (Fig. S3C). Since the particles are crystalline (Fig. 2D), the contrast changes result from changes in particle orientation, and the correspondingly different diffraction intensities ${ }^{17}$. It was observed that the particle rotates along the direction of movement (rolling) for the $15 \mathrm{~nm}$ particle. The rolling motion suggests a significant effect of the substrate surface on the particle movement ${ }^{18,19}$. Nanoparticles in solution can be weakly bound near the surface due to a potential for attraction between the surface and the particles ${ }^{20}$. Luedtke and Landman ${ }^{21}$ predicted this type of anomalous diffusion behavior for nanoparticles near a dry-surface about ten years ago, but it has not been possible to directly observe this previously. In their model, the initiation of rolling is attributed to a thermal fluctuation overcoming the energy barrier of interaction between the substrate and the particle. The differences in our case are that the particles are in a thin film liquid and only limited rolling distances were observed. Statistically, the step lengths consist roughly of two normal distributions (Fig. 1D) instead of Lévy flight characteristics (a power law dependence of step length ${ }^{21-23}$ ). Experimentally, these two modes of particle motion can be classified as the motion in the liquid thin film and the motion confined on the substrate surface. There also might be additional factors (e.g. lateral capillary forces or local convective flow) from the liquid 
surface affecting the movement of the larger particles more strongly than the smaller particles $^{24}$. In a thin liquid film, such effects can drive the larger particles to move faster than the smaller ones. We have found that the jumps of the largest particles are roughly along certain directions (Fig. 1A), which suggests that the particles might be moving in the direction of local convective flow or are dragged by a lateral capillary force. Our observation is consistent with earlier studies on the size-dependent separation of colloidal nanoparticles during fluid evaporation ${ }^{25-26}$. However, direct observation of the sizedependent movement of individual nanoparticles during fluid evaporation has not been possible before.

Particle orientation changes correlated to particle jumps in the liquid film are more clearly seen in the motions of an asymmetric particle $(5 \times 10 \mathrm{~nm})$. For example, rotation from vertical to in-plane and rolling around its long axis in addition to in-plane rotation and translation were observed, see Fig. $3 \mathrm{~A}$ and movie $2 \mathrm{~S}$. The consecutive center-of-mass translational motion and orientation were plotted in Fig. 3B, in which orientations $\theta$ relative to the $x$-axis are labeled with a rainbow color scale. A uniaxial anisotropic particle is characterized by parallel and transverse components of hydrodynamic friction coefficients, $\gamma_{/ /}$and $\gamma_{\perp}$ respectively, for motion parallel to its long axis $\left(X_{/ /}\right)$and perpendicular to its long axis $\left(Y_{\perp}\right)$. In general, $\gamma_{/ /}$is smaller than $\gamma_{\perp}{ }^{27}$, and consequently a larger diffusion coefficient along $X_{/ /}$axis than along $Y_{\perp}$ axis is expected if a particle's rotation is prohibited. Such anisotropic motion is also valid for a short time when rotation is allowed ${ }^{28}$. We resolved this behavior by decomposing the rod's displacement into components relative to the body frame $\left[X_{/ /}, Y_{\perp}\right]$ or the lab frame $[x, y, \theta]$. As shown in Fig. 3C, their relation can be expressed as $X_{/ /}=\Delta x \cos \theta+\Delta y \sin \theta$ and 
$Y_{\perp}=-\Delta x \sin \theta+\Delta y \cos \theta$, where $\Delta x=\left(x_{1}-x_{2}\right), \Delta y=\left(y_{1}-y_{2}\right)$ and $\theta=\theta_{1}$. We calculated the mean-square displacement (MSD) vs time $(t)$ along $X_{/ /}$and $Y_{\perp}$ axes (Fig. 3D) and obtained the anisotropic diffusion coefficients of $0.26 \mathrm{~nm}^{2} / \mathrm{s}$ along $X_{/ /}$axis and 0.16 $\mathrm{nm}^{2} / \mathrm{s}$ along $Y_{\perp}$ axis (estimated from the slope of the plots). The histograms of the displacement distribution along the two axes show a larger deviation corresponding to a larger diffusion coefficient along the $X_{/ /}$axis (Fig. 3E). The detailed evolution of the rod trajectory from short-term anisotropic motion to long-time isotropic motion due to the rod rotation can be further resolved.

When following the behavior of individual particles before and after the initiation of drying patches, we found distinctly different modes of motion. This is apparent from the trajectory of a $5 \mathrm{~nm}$ spherical particle motion through a sequence of movements in Fig. 4A. The displacement $(\lambda)$ during a time interval $(\Delta t) v s$ time $(t)$ was analyzed (Fig. 4B) using the same method as in Fig. 2B. Similar behavior of the particle jumps followed by small-step movements was observed during the first $400 \mathrm{~s}$ of movement. Jumps corresponding to particle rolling are observed.

At the later stages of the particle movement (see Fig. 4B), the motion is heavily biased. In addition, large-step displacements (also manifested as jumps in the trajectory, see Fig. $4 \mathrm{~A} b \rightarrow \mathrm{c}$ ) were an order of magnitude larger than the average jump-length in a liquid film at the early stage. The corresponding images elucidate that drying patches formed in the liquid film and particles were dragged by the nearby retracting liquid front (Fig. 4C). Since the particle contrast does not change during these drying patch induced large jumps, we conclude that the motion proceeded primarily by sliding at this stage. 
Correlated jumps between nearby particles were observed in some cases, supporting the suggestion of liquid drag.

In the present study we have directly resolved the complex motion of inorganic nanoparticles in a liquid thin film during solvent evaporation. Our observations reveal three distinctly different modes of particle motion: (1) center-of-mass displacement over short length scales, (2) rolling over longer length scales, and finally, (3) dragging by the fluid front over considerably longer distances. A combination of these three modes of movements determines the ultimate motion of the particle during the drying process. This work has provided a unique view of the motion of individual nanoparticles during solvent evaporation, providing the necessary groundwork for future studies of correlated motion in more concentrated particle solutions and for studies of particle diffusion during selfassembly processes intended to create complex functional nanoparticle arrangements. In addition, gold nanoparticles have been used as labels for electron microscopy of frozen biological samples for decades. The work described here suggests that it may not be long before dynamical motion of biological molecules can be tracked by electron microscopy in physiological environments.

\section{Acknowledgement}

We gratefully acknowledge J. Ku, Professor P. L. Geissler and Professor H. Yang for useful discussions, Dr. H. Liu and J. Turner for their help at the beginning of image processing and data analysis. S. Claridge is supported by an NSF-IGERT predoctoral fellowship. This work was performed at the National Center for Electron Microscopy, Lawrence Berkeley National Laboratory, and was supported by the Office of Science, 
Office of Basic Energy Sciences of the U.S. Department of Energy under Contract No. DE-AC02-05CH11231.

\section{$\underline{\text { Supporting Information }}$}

Liquid cell fabrication, experimental details and discussions on electron beam effects are available free of charge via the Internet at http://pubs.acs.org.

\section{References}

1. Deegan, R. D.; Bakajin, O.; Dupont, T. F.; Huber, G.; Nagel, S. R.; Witten, T. A. Nature 1997, 389, (6653), 827-829.

2. Rabani, E.; Reichman, D. R.; Geissler, P. L.; Brus, L. E. Nature 2003, 426, (6964), 271-274.

3. Bigioni, T. P.; Lin, X. M.; Nguyen, T. T.; Corwin, E. I.; Witten, T. A.; Jaeger, H. M. Nat. Mater. 2006, 5, (4), 265-270.

4. Cui, Y.; Bjork, M. T.; Liddle, J. A.; Sonnichsen, C.; Boussert, B.; Alivisatos, A. P. Nano Lett. 2004, 4, (6), 1093-1098.

5. Lin, X. M.; Jaeger, H. M.; Sorensen, C. M.; Klabunde, K. J. J. Phys. Chem. B 2001, 105, (17), 3353-3357.

6. Granick, S. Science 1991, 253, (5026), 1374-1379.

7. Heuberger, M.; Zach, M.; Spencer, N. D. Science 2001, 292, (5518), 905-908.

8. Kaizuka, Y.; Groves, J. T. Phys. Rev. Lett. 2006, 96, (11), 118101.

9. Froltsov, V. A.; Klapp, S. H. L. J. Chem. Phys. 2006, 124, (13), 134701.

10. Myers, T. G. Siam Rev. 1998, 40, (3), 441-462. 
11. Williamson, M. J.; Tromp, R. M.; Vereecken, P. M.; Hull, R.; Ross, F. M. Nat. Mater. 2003, 2, (8), 532-536.

12. Howe, J. M.; Yokota, T.; Murayama, M. J. Electron Microsc. 2004, 53, (2), $107-$ 114.

13. Yokota, T.; Murayama, M.; Howe, J. M. Phys. Rev. Lett. 2003, 91, (26), 265504.

14. Egerton, R. F.; Li, P.; Malac, M. Micron 2004, 35, (6), 399-409.

15. Chen, C. T.; Tseng, F. G.; Chieng, C. C. Sens. Actuators A: Phys. 2006, 130, 1219.

16. Raptis, T. E.; Raptis, V. E.; Samios, J. J. Phys. Chem. B 2007, 111, (49), 1368313693.

17. Fultz, B.; Howe, J. M. Transmission Electron Microscopy and Diffractometry of Materials, Springer: Berlin, 2002.

18. Bardotti, L.; Jensen, P.; Hoareau, A.; Treilleux, M.; Cabaud, B.; Perez, A.; Aires, F. C. S. Surf. Sci. 1996, 367, (3), 276-292.

19. Naumovets, A. G.; Zhang, Z. Y. Surf. Sci. 2002, 500, (1-3), 414-436.

20. Sonnichsen, C.; Alivisatos, A. P. Nano Lett. 2005, 5, (2), 301-304.

21. Luedtke, W. D.; Landman, U. Phys. Rev. Lett. 1999, 82, (19), 3835-3838.

22. Shlesinger, M. F.; Zaslavsky, G. M.; Klafter, J. Nature 1993, 363, (6424), 31-37.

23. Chechkin, A. V.; Klafter, J.; Gonchar, V. Y.; Metzler, R.; Tanatarov, L. V. Phys. Rev. E 2003, 67, (1), 010102.

24. Lopez, M.; Graham, M. D. Phys. Fluids 2007, 19, (7), 073602.

25. Yamaki, M.; Higo, J.; Nagayama, K. Langmuir 1995, 11, (8), 2975-2978.

26. Bestehorn, M.; Neuffer, K. Phys. Rev. Lett. 2001, 87, (4), 046101. 
27. Happel, J.; Brenner, H. Low Reynolds Number Hydrodynamics, Kluwer and Dordrecht: Netherlands, 1991.

28. Han, Y.; Alsayed, A. M.; Nobili, M.; Zhang, J.; Lubensky, T. C.; Yodh, A. G. Science 2006, 314, (5799), 626-630. 
Figures:

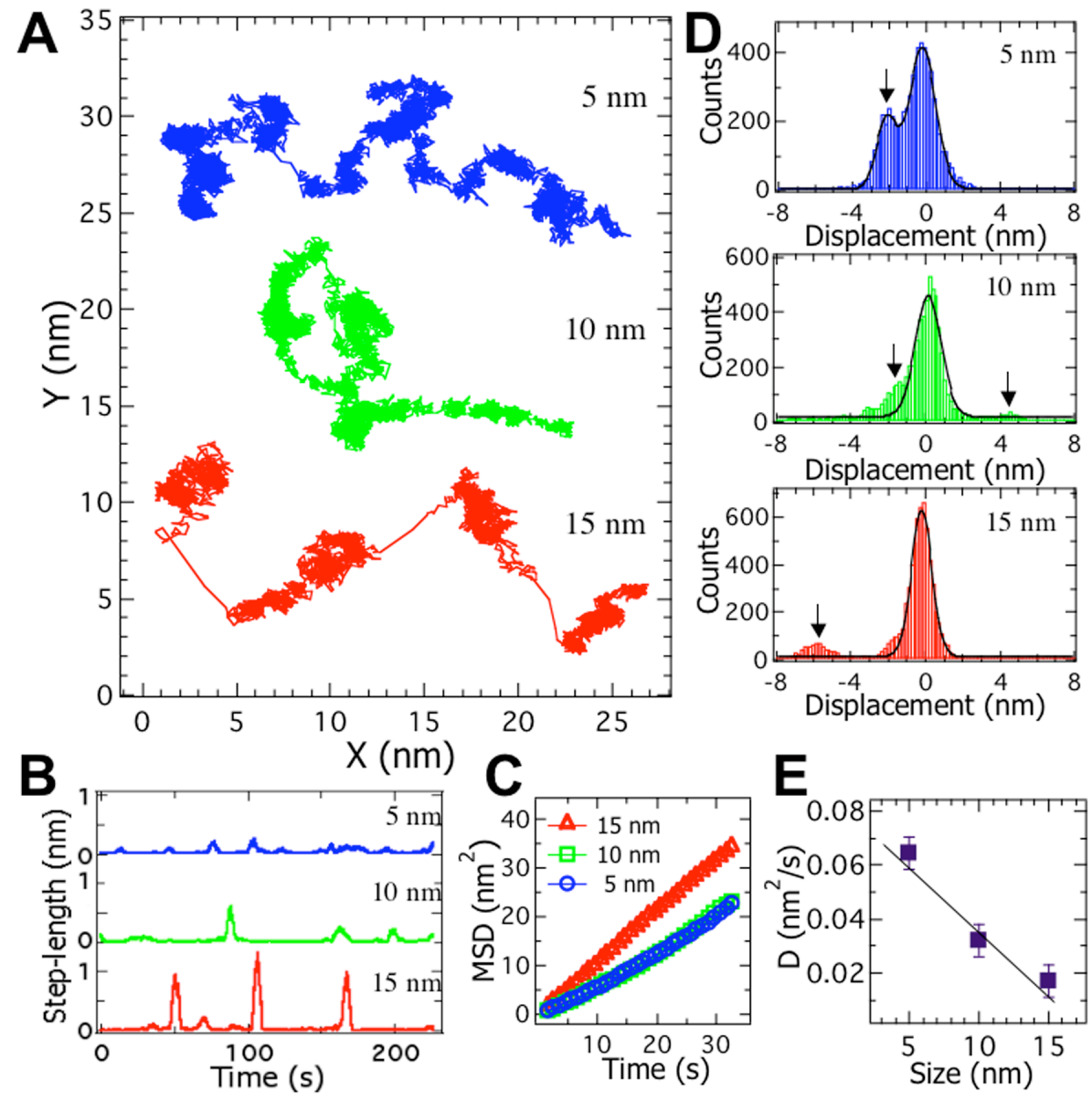

Fig. 1. Analysis of different sized particle motion. (A) Trajectories of 5, 10 and $15 \mathrm{~nm}$ particle motion in the same liquid film recorded over the same time period of $233 \mathrm{~s}$. Initial positions are arbitrary. (B) Displacements during a time interval of $6 \mathrm{~s}$ vs time. (C) Mean-square displacement, $M S D$, vs time. (D) Histograms showing the distributions of different sized particle displacements during a time interval of $6 \mathrm{~s}$. Black curves show Gaussian fits. Additional peaks due to the larger step movements are marked by arrows. (E) Diffusion constants, $D$, due to the small-step movements only (corresponding to the main peak around zero displacement in histograms) vs particle size. Black line show linear fit. 


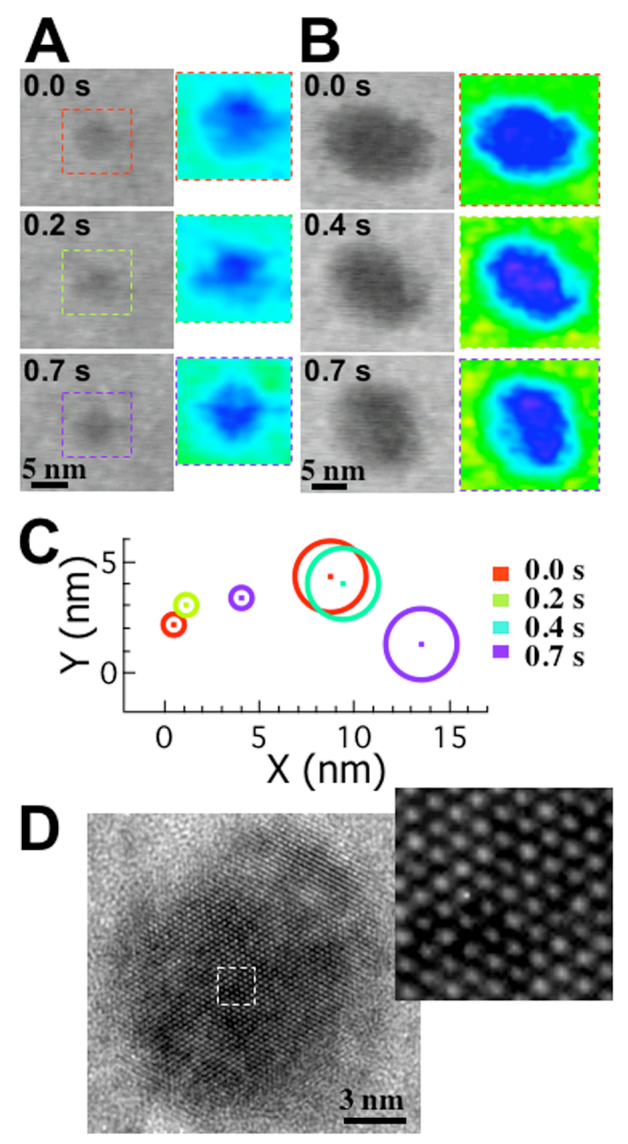

Fig. 2. Jump motion corresponding to particle orientation changes. (A) An image sequence showing the orientation changes of a $5 \mathrm{~nm}$ particle during a jump. The orientation changes indicated by the difference in diffraction intensities within the particle are highlighted using color gradient maps. (B) An image sequence showing the orientation changes of a $15 \mathrm{~nm}$ particle during a jump. (C) Jump distances (center-ofmass displacements) of the $5 \mathrm{~nm}$ particle in (A) and $15 \mathrm{~nm}$ particle in (B) within a time interval of $0.7 \mathrm{~s}$. (D) High resolution TEM image of a gold particle inside the liquid cell after the diffusion experiment (the liquid has dried out). An enlarged view of the marked section is shown in the upper right. The original video images corresponding to (A) and (B) are provided in Fig. S3 (A) and (B). 


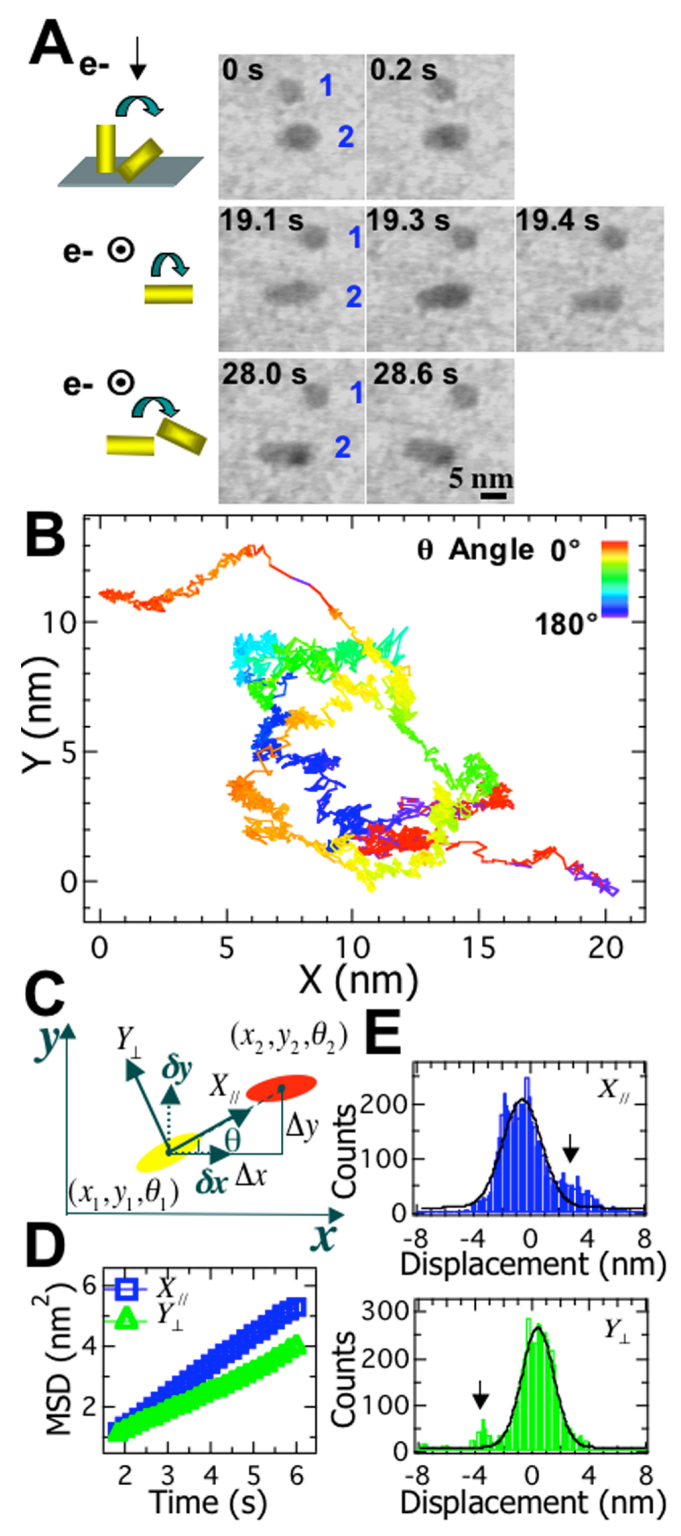

Fig. 3. Asymmetric particle motion. (A) Selected image sequences showing a $510 \mathrm{~nm}$ asymmetric particle (particle 2) undergoing different types of motion: rotation from vertical to in-plane, rolling around its long-axis and wagging. Particle 1 is a reference particle with no random motion. The direction of the electron beam (e-) is indicated by an arrow; a cirle indicates that the beam is normal to the figure. (B) A trajectory of the rodshaped particle's 5,550-step center-of-mass displacements in a liquid thin film. Each step is $1 / 30 \mathrm{~s}$. Orientations are labeled with a rainbow color scale. (C) Particle motion can be referred to the body frame (,) or the lab frame $(x, y$, ). (D) Mean-square displacement, $M S D$, vs time showing the asymmetric motion along and axes. (E) Histograms showing the distributions of the particle displacements during a time interval of $6 \mathrm{~s}$ along and axes. Black curves show Gaussian fits. Additional peaks due to the larger step movements are marked by arrows. 


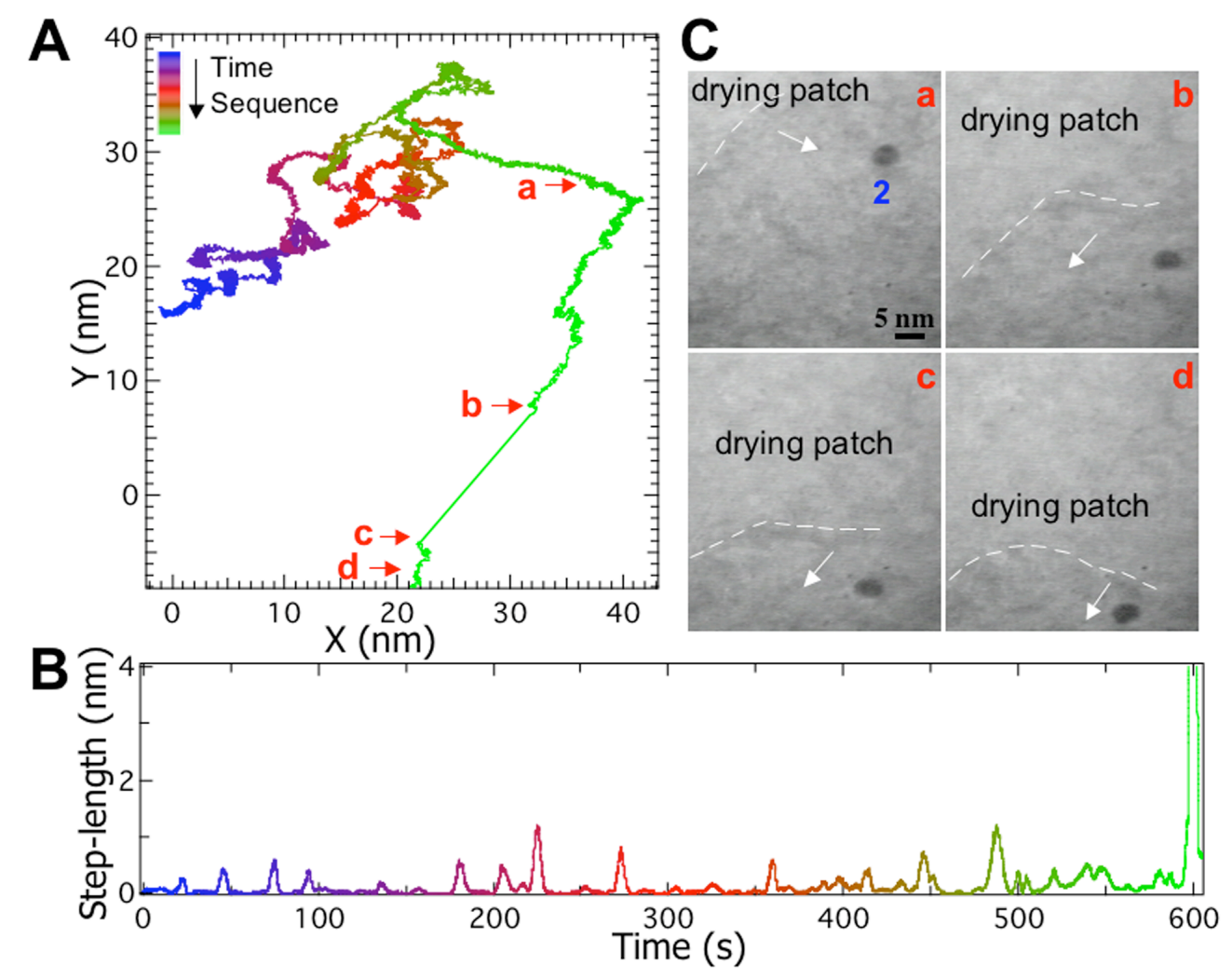

Fig. 4. A $5 \mathrm{~nm}$ particle motion in a liquid thin film until the formation of drying patches. (A) Trajectory of 18,150 -step movement. Each step is $1 / 30 \mathrm{~s}$. The initial position is arbitrary. The time variable is labeled with a rainbow color scale. (B) Displacement during a time interval of $2 \mathrm{~s} v s$ time. (C) An image sequence corresponding to the positions in the trajectory in (A) showing the particle being dragged by the nearby retracting liquid front at the later stage of solvent evaporation. a, $602.0 \mathrm{~s} ; \mathrm{b}, 665.9 \mathrm{~s} \mathrm{c}$, $666.0 \mathrm{~s}$ and $\mathrm{d}, 671.6 \mathrm{~s}$. 
DISCLAIMER: This document was prepared as an account of work sponsored by the United States Government. While this document is believed to contain correct information, neither the United States Government nor any agency thereof, nor The Regents of the University of California, nor any of their employees, makes any warranty, express or implied, or assumes any legal responsibility for the accuracy, completeness, or usefulness of any information, apparatus, product, or process disclosed, or represents that its use would not infringe privately owned rights. Reference herein to any specific commercial product, process, or service by its trade name, trademark, manufacturer, or otherwise, does not necessarily constitute or imply its endorsement, recommendation, or favoring by the United States Government or any agency thereof, or The Regents of the University of California. The views and opinions of authors expressed herein do not necessarily state or reflect those of the United States Government or any agency thereof or The Regents of the University of California. 